\title{
Shielding and synchrotron radiation in toroidal waveguide
}

\author{
G. V. Stupakov \\ Stanford Linear Accelerator Center, Stanford University, Stanford, California 94309
}

I. A. Kotelnikov

Budker Institute of Nuclear Physics, Lavrent'ev Prospect 11, Novosibirsk, 630090, Russia

(Received 6 January 2003; published 3 March 2003)

\begin{abstract}
We develop a new approach to the calculation of the synchrotron radiation in a toroidal vacuum chamber. Using a small parameter $\epsilon=\sqrt{a / R}$, where $a$ is the characteristic size of the cross section of the toroid and $R$ is the bending radius, we simplify Maxwell's equations assuming that the characteristic frequency of the modes $\omega \sim c / a \epsilon$ and neglect terms of higher order in $\epsilon$. For a rectangular cross section of the waveguide, we find an analytical solution of the equations and analyze their asymptotics at very high frequency. We then obtain an equation which gives radiation into each synchronous mode. We demonstrate the flexibility of the new method by calculating the frequencies and the loss factors for the lowest modes in square and round waveguides.
\end{abstract}

DOI: 10.1103/PhysRevSTAB.6.034401

PACS numbers: 29.27. Bd, 41.75.Ht

\section{INTRODUCTION}

For short bunches of high-energy electrons or positrons, typical for modern accelerators and photon sources, one of the important elements of the beam dynamics is the effect of coherent synchrotron radiation (CSR) of the beam [1]. In vacuum, if the reduced wavelength of the radiation exceeds the bunch length, the beam radiates coherently, and the power of the radiation increases many orders of magnitude relative to the incoherent radiation at the same wavelength. Typically, however, CSR at such wavelengths is suppressed due to the shielding effect of the conducting walls of the vacuum chamber. CSR at shorter wavelengths can still play a role in a microwave instability causing microbunching of the beam [2].

Synchrotron radiation of a relativistic beam moving in a toroidal chamber with conducting walls has been extensively studied in the past (see, e.g., [3-5]). Many important features of the radiation have been analyzed based on the direct solution of the Maxwell equations for rectangular cross section of the chamber, in the limit of large values of the azimuthal number $n$. In the particular case of the parallel conducting plates, the method of image charges was used in Ref. [6]. Unfortunately, these methods can be used only for a rectangular cross section of the chamber and are not applicable to other shapes encountered in practice.

In this paper, we study radiation of a relativistic particle moving in a circular orbit inside of a toroidal chamber, using a different approach. We introduce a small parameter $\epsilon$ equal to $\sqrt{a / R}$, where $a$ is the characteristic chamber size and $R$ is the toroid radius. A relativistic particle circulating inside the toroid can resonantly excite the synchronous electromagnetic modes that have phase velocity equal to the particle's velocity $v \approx c$. As discussed in Refs. [3,4], for a given toroid, the synchronous modes have wave numbers $k$ greater than a minimal value $k_{\min }=\omega_{\min } / c$ :

$$
k \geqslant \frac{\omega_{\min }}{c} \sim \frac{R^{1 / 2}}{a^{3 / 2}} \sim \frac{1}{\epsilon a} \gg a^{-1} .
$$

Using the scaling $k a \sim \epsilon^{-1}$ that follows from Eq. (1) and considering only modes that have phase velocity close to the speed of light, we simplify Maxwell's equations, keeping only terms of the lowest order in $\epsilon$. We then solve these equations for the synchronous modes and find the intensity of the particle's radiation into each mode.

The advantage of this approach is that instead of dealing with a solution of a full set of Maxwell's equations for a chosen geometry of the toroidal chamber, we first simplify the equations using an appropriate scaling of the relevant parameters of the problem (such as $k, R$, and $a$ ). The simplified equations do not contain the small parameter $\epsilon$. In this paper, we focus on two casesrectangular and round cross sections of the toroid. The first case allows an analytical solution for the eigenmodes and permits a detailed comparison with the results known in the literature. The second case relies on a numerical solution of the equations and demonstrates applicability of the new method to the cross sections with the shapes other than the rectangular one.

Throughout the paper we assume perfect conductivity of the walls. We also consider relativistic particles with the Lorentz factor $\gamma \gg 1$.

The paper is organized as follows. In Sec. II we simplify Maxwell's equations in the limit of small $\epsilon$, and in Sec. III we formulate boundary conditions for the equations. A variational principle for the equations is derived in Appendix A. In Sec. IV we obtain an analytical solution for the rectangular cross section of the toroid. In Sec. $\mathrm{V}$ we present expressions for the energy radiated into synchronous modes by a moving charge and introduce 
the impedance associated with those modes and the longitudinal wake. The derivation of the radiative energy loss is given in Appendix B. In Sec. VI we present results for numerically computed parameters of the lowest modes for the cases of square and round cross sections. In Appendix $\mathrm{C}$ we derive a formula for the group velocity of toroidal modes. In Appendix D we consider a limit of high frequency and show that our results in this limit reduce to the standard formulas for the synchrotron radiation in free space.

\section{EQUATIONS FOR ELECTROMAGNETIC FIELD}

In this section we will derive equations for the electric and magnetic fields in the toroidal waveguide. We consider a smooth toroidal vacuum chamber of radius $R$ and arbitrary cross section. The geometry of the problem and the choice of the coordinate system are shown in Fig. 1. We use the cylindrical coordinate system $r, \theta, z$ and the notation $x$ for the difference $x=r-R$.

We assume that all components of the field have the following dependence on time and the azimuthal angle $\theta$ :

$$
\boldsymbol{E}, \boldsymbol{H} \propto e^{-i \omega t+i k R \theta} .
$$

We are interested in such solutions of Maxwell's equations which have the phase velocity equal to the velocity of the particle

$$
\frac{\omega}{k}=v \approx c\left(1-\frac{1}{2 \gamma^{2}}\right)
$$

To keep track of the order of magnitudes, we will assume that $x$ and $z$ coordinates are of the order of $\epsilon^{0} \sim$ 1 and the orbit radius $R$ is of the order of $\epsilon^{-2}$, where $\epsilon^{2} \sim$ $a / R$ is a formal small parameter of the problem. According to Eq. (1), $k$ is assigned the order of $\epsilon^{-1}$. We will also assume that $\gamma \sim \epsilon^{-1}$. As we will see below, if the transverse to the orbit components of the fields $E_{r}, E_{z}$, $H_{r}$, and $H_{z}$ are of the order of 1, then the longitudinal components $E_{\theta}, H_{\theta} \sim \epsilon$. The latter can be explained by the fact that in the limit of high frequencies which we consider here the effect of the walls is relatively small, and the electromagnetic waves are almost transverse relative to the direction of the propagation $\theta$.

From the Maxwell equations

$$
\frac{i \omega}{c} H_{r}=\frac{i k R}{r} E_{z}-\frac{\partial E_{\theta}}{\partial z}, \quad \frac{-i \omega}{c} E_{r}=\frac{i k R}{r} H_{z}-\frac{\partial H_{\theta}}{\partial z},
$$

using Eq. (3) and the smallness of $E_{\theta}$ and $H_{\theta}$ we find to the lowest order

$$
H_{r}=E_{z}, \quad H_{z}=-E_{r} .
$$

These are relations that hold in a plane electromagnetic wave; in our case they are satisfied approximately to order $\epsilon^{2}$.

From the other pair of Maxwell equations

$$
-i \frac{\omega}{c} H_{\theta}=\frac{1}{r} \frac{\partial r E_{z}}{\partial r}-\frac{\partial E_{r}}{\partial z}, \quad i \frac{\omega}{c} E_{\theta}=\frac{1}{r} \frac{\partial r H_{z}}{\partial r}-\frac{\partial H_{r}}{\partial z},
$$

using Eqs. (5) we express $H_{\theta}$ and $E_{\theta}$ in terms of $E_{r}$ and $E_{z}$

$$
H_{\theta}=\frac{i}{k}\left(\frac{\partial E_{z}}{\partial x}-\frac{\partial E_{r}}{\partial z}\right), \quad E_{\theta}=\frac{i}{k}\left(\frac{\partial E_{r}}{\partial x}+\frac{\partial E_{z}}{\partial z}\right),
$$

where again we neglected terms of the order of $\epsilon^{3}$.

Having expressed $E_{\theta}, H_{z}, H_{\theta}$, and $H_{r}$ in terms of $E_{r}$ and $E_{z}$, we can now derive equations for $E_{r}, E_{z}$ if we note that $E_{z}$ and $H_{z}$ (and hence $E_{r}$ ) satisfy the wave equation

$$
\Delta E_{z}+\frac{\omega^{2}}{c^{2}} E_{z}=0, \quad \Delta E_{r}+\frac{\omega^{2}}{c^{2}} E_{r}=0
$$

Let us write the first of Eqs. (8) as

$$
\frac{1}{r} \frac{\partial}{\partial r} r \frac{\partial E_{z}}{\partial r}+\frac{\partial^{2} E_{z}}{\partial z^{2}}+\left(\frac{\omega^{2}}{c^{2}}-\frac{k^{2} R^{2}}{r^{2}}\right) E_{z}=0
$$

The same equation holds for $E_{r}$. Substituting $r=R+x$, expanding in small ratio $x / R$ and using Eq. (3), we find

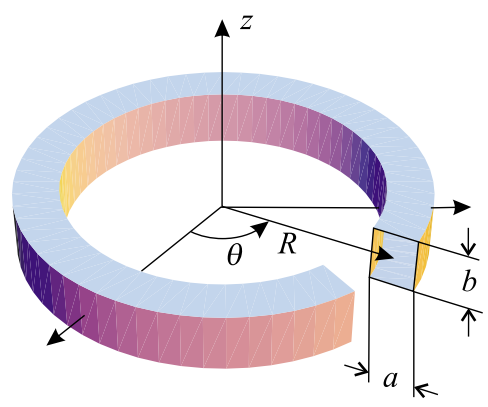

FIG. 1. (Color) Smooth toroidal vacuum camera for circular (left) and rectangular (right) cross sections with $R$ the major radius of the toroid. It is assumed that a particle's orbit goes through the center of the toroidal chamber and has the same radius $R$. A segment is removed for illustration purposes. 


$$
\begin{aligned}
& \Delta_{\perp} E_{z}+2 k^{2}\left(\Lambda+\frac{x}{R}\right) E_{z}=0, \\
& \Delta_{\perp} E_{r}+2 k^{2}\left(\Lambda+\frac{x}{R}\right) E_{r}=0,
\end{aligned}
$$

where $\Delta_{\perp}=\partial^{2} / \partial x^{2}+\partial^{2} / \partial z^{2}$,

$$
\Lambda(\omega, k)=\frac{1}{2}\left(\frac{\omega^{2}}{k^{2} c^{2}}-1\right) .
$$

The parameter $\Lambda$ measures deviation of the phase velocity $\omega / k$ from the speed of light $c$; in what follows we will assume that $\Lambda \sim \epsilon^{2}$. Note that in derivation of Eq. (5) we set $\omega=c k$ which formally corresponds to $\Lambda=0$; it is easy to check, however, that the equations are still valid for $\Lambda \sim \epsilon^{2}$, the error being of the order of $\epsilon^{2}$.

Although we have separate Eqs. (10) for $E_{r}$ and $E_{z}$, in the next section we will see that the functions $E_{r}, E_{z}$ are coupled through the boundary conditions on the conducting wall.

\section{BOUNDARY CONDITIONS FOR $E_{R}, E_{Z}$}

Let $\mathbf{n}=\left(n_{x}, n_{z}\right)$ be the normal vector to the toroid's surface in the plane $x z$ directed toward the metal, while $\tau=\left(-n_{z}, n_{x}\right)$ be the vector tangential to the surface in the same plane. For the perfectly conducting wall, the tangential electric field vanishes at the surface: $E_{\theta}=$ $E_{\tau}=0$. The first equality, $E_{\theta}=0$, yields

$$
\frac{\partial E_{z}}{\partial z}+\frac{\partial E_{r}}{\partial x}=0
$$

The second one gives

$$
n_{x} E_{z}-n_{z} E_{r}=0 .
$$

In the general case, these boundary conditions couple Eqs. (10) so that one has to solve them together. The two equations with the boundary conditions constitute an eigenvalue problem - for a given $k$, the solution exists only for a discreet set of values of $\omega$. If, however, we specify the value of $\Lambda$ (e.g., $\Lambda=0$, corresponding to waves propagating with the phase velocity equal to $c$ ), then the solution exists only for a discreet set of eigenvalues $k$. Note that both the equations and the boundary conditions do not involve complex numbers - this means that the field components $E_{r}$ and $E_{z}$ of eigenmodes can always be chosen real. From Eqs. (5) and (7) it follows that $H_{r}$ and $H_{z}$ are also real, and $E_{\theta}$ and $H_{\theta}$ are purely imaginary.

Equations (10) with the boundary conditions Eqs. (12a) and (12b) can also be formulated as a variational problem which can be used for the numerical solution of the problem. We derive the corresponding variational functional in Appendix A.

\section{A. Rectangular toroid}

For a rectangular cross section of the toroidal camera with the width $a$ the size along the horizontal axis $x$ (directed along the major radius $R$ of the toroid), and the height $b$ the size along the vertical axis $z$ (see Fig. 1), it is readily seen that the boundary conditions for the $E_{r}$ and $E_{z}$ functions separate. Noting that $n_{x}= \pm 1, n_{z}=0$ at $x= \pm a / 2$ while $n_{x}=0, n_{z}= \pm 1$ at $z= \pm b / 2$, we find

$$
\frac{\partial E_{r}}{\partial x}=0 \text { at } x= \pm a / 2, \quad E_{r}=0 \text { at } z= \pm b / 2
$$

for the $E_{r}$ component, and

$$
E_{z}=0 \text { at } x= \pm a / 2, \quad \frac{\partial E_{z}}{\partial z}=0 \text { at } z= \pm b / 2
$$

for $E_{z}$.

\section{B. Round toroid}

For the toroid with a circular cross section of radius $a$ we introduce the poloidal angle $\phi$ and the radius vector $\rho$, such that $x=\rho \cos \phi, z=\rho \sin \phi$. We then have $\partial / \partial n=$ $\partial / \partial \rho, n_{x}=\cos \phi$, and $n_{z}=\sin \phi$. The boundary condition (12a) at $\rho=a$ yields

$$
\frac{1}{\rho} \frac{\partial \rho E_{r}}{\partial \rho} \cos \phi+\frac{1}{\rho} \frac{\partial \rho E_{z}}{\partial \rho} \sin \phi=0 .
$$

The other boundary condition $(12 b)$ reduces to

$$
E_{z} \cos \phi-E_{r} \sin \phi=0 .
$$

\section{ANALYTICAL SOLUTION FOR RECTANGULAR TOROID}

As was pointed out above, in the case of a rectangular toroid Eqs. (10) and the boundary conditions decouple and can be solved separately for $E_{r}$ and $E_{z}$. This means that there are two sets of modes with different polarization: one in which $E_{r}=0$ and the other with $E_{z}=0$. We will call the first set $E_{z}$ modes, or vertically polarized, and the second one $E_{r}$ modes, or horizontally polarized. Separating $x$ and $z$ variables and using the boundary conditions (13a) and (13b) at $z= \pm b / 2$ we find

$$
E_{r}(x, z)=E_{r}(x) \sin \left[\frac{\pi p}{b}\left(z+\frac{b}{2}\right)\right], \quad p=1,2,3, \ldots
$$

for the $E_{r}$ modes, and

$$
E_{z}(x, z)=E_{z}(x) \cos \left[\frac{\pi p}{b}\left(z+\frac{b}{2}\right)\right], \quad p=0,1,2, \ldots
$$

for the $E_{z}$ modes. 
In what follows, we will use the notation $U(x)$ both for $E_{r}(x)$ and $E_{z}(x)$ when it is not important to distinguish between them. The equation for $U$ takes the form

$$
\frac{d^{2} U}{d x^{2}}+2 k^{2}\left(\frac{x}{R}+\Lambda-\frac{\pi^{2} p^{2}}{2 k^{2} b^{2}}\right) U=0 .
$$

To proceed further we introduce the dimensionless coordinate

$$
\xi=x\left(\frac{2 k^{2}}{R}\right)^{1 / 3}
$$

and dimensionless parameters $\xi_{0}$ and $\xi_{a}$

$$
\xi_{0}=\frac{\pi^{2} p^{2} R^{2 / 3}}{\left(2 k^{2}\right)^{2 / 3} b^{2}}-\left(2 k^{2} R^{2}\right)^{1 / 3} \Lambda, \quad \xi_{a}=a\left(\frac{2 k^{2}}{R}\right)^{1 / 3} .
$$

This yields the equation

$$
\frac{d^{2} U}{d \xi^{2}}+\left(\xi-\xi_{0}\right) U=0
$$

The boundary conditions at $\xi= \pm \xi_{a} / 2 \mathrm{read}$

$$
U(\xi)=0,
$$

for the $E_{z}$ wave, and

$$
\frac{d}{d \xi} U(\xi)=0
$$

for the $E_{r}$ wave.

The general solution of Eq. (17) involves the Airy functions $\mathrm{Ai}$ and $\mathrm{Bi}$ :

$$
U(\xi)=C_{1} \operatorname{Ai}\left(\xi_{0}-\xi\right)+C_{2} \operatorname{Bi}\left(\xi_{0}-\xi\right),
$$

where $C_{1,2}$ are unknown constants to be found from the boundary conditions. Boundary conditions for $E_{r}$ yield the dispersion equation

$$
\begin{aligned}
& \operatorname{Ai}^{\prime}\left(\xi_{0}-\xi_{a} / 2\right) \mathrm{Bi}^{\prime}\left(\xi_{0}+\xi_{a} / 2\right) \\
& \quad=\operatorname{Ai}^{\prime}\left(\xi_{0}+\xi_{a} / 2\right) \mathrm{Bi}^{\prime}\left(\xi_{0}-\xi_{a} / 2\right),
\end{aligned}
$$

and the boundary conditions for $E_{z}$ yield

$$
\begin{aligned}
& \operatorname{Ai}\left(\xi_{0}-\xi_{a} / 2\right) \operatorname{Bi}\left(\xi_{0}+\xi_{a} / 2\right) \\
& \quad=\operatorname{Ai}\left(\xi_{0}+\xi_{a} / 2\right) \operatorname{Bi}\left(\xi_{0}-\xi_{a} / 2\right) .
\end{aligned}
$$

We will mark a sequence of eigenvalues of Eqs. (19a) and (19b) by an integer index $m$. This index is defined so as to indicate the number of nodes in the function $U(\xi)$ on the interval $-\xi_{a} / 2 \leqslant \xi<\xi_{a} / 2$. For the $E_{r}$ modes, $m$ takes values of $0,1,2, \ldots$. For the $E_{z}$ modes, due to the boundary condition $U\left(-\xi_{a} / 2\right)=0$, the values of $m$ are $1,2,3, \ldots$.

Let us consider the case when $\Lambda=0$, that is, the waves that have the phase velocity equal to the speed of light. Note that, in this case,

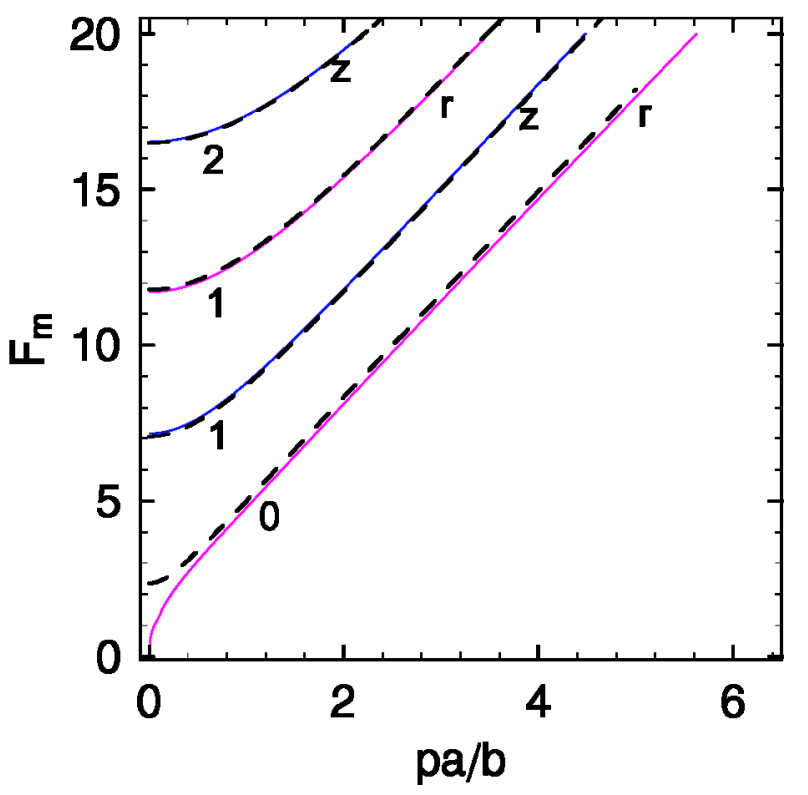

FIG. 2. (Color) Solution of the dispersion equation $\Lambda=0$ for various mode numbers $m$ indicated on the plot. $E_{r}$ mode: magenta (also indicated by symbol $r$ ); $E_{z}$ mode: blue (indicated by symbol $z$ ). The dashed lines show the approximate solutions discussed in the subsection of IV.

$$
\xi_{0}=\frac{\pi^{2}}{\xi_{a}^{2}}\left(\frac{p a}{b}\right)^{2}
$$

and hence from the dispersion Eqs. (19a) and (19b) one can find the relation between $\xi_{a}$ and the parameter $p a / b$. The latter can be expressed as

$$
k=\left(\frac{R}{a^{3}}\right)^{1 / 2} F_{m}\left(\frac{p a}{b}\right),
$$

where $F_{m}$ is a function defined from the solution of Eqs. (19a) and (19b). The plot of this function for both $E_{r}$ and $E_{z}$ modes for a few lowest radial mode numbers $m$ is shown in Fig. 2 by solid lines. From this figure, one can see that the minimal frequency in a rectangular toroidal waveguide is attained for the $E_{r}$ mode with $m=0$. For example, in the case of a square cross section, $a=b$, this minimal frequency is $\omega_{\min }=4.78 c R^{1 / 2} a^{-3 / 2}$. Note that $k_{\min }=\omega_{\min } / c$ confirms the estimate used in Eq. (1).

\section{Limit of High Frequency, $\omega \gg \omega_{\min }$}

In the limit of high frequencies, $\omega \gg \omega_{\min } \sim$ $c R^{1 / 2} a^{-3 / 2}$, the exact solution for the rectangular cross section can be considerably simplified. In this limit, as we will show below,

$$
\xi_{0}+\xi_{a} / 2 \gg 1
$$

and the factors $\operatorname{Ai}\left(\xi_{0}+\xi_{a} / 2\right) \operatorname{Bi}\left(\xi_{0}-\xi_{a} / 2\right) / \operatorname{Bi}\left(\xi_{0}+\right.$ $\left.\xi_{a} / 2\right)$ and $\mathrm{Ai}^{\prime}\left(\xi_{0}+\xi_{a} / 2\right) \mathrm{Bi}^{\prime}\left(\xi_{0}-\xi_{a} / 2\right) / \mathrm{Bi}^{\prime}\left(\xi_{0}+\xi_{a} / 2\right)$ in the dispersion relations (19a) and (19b) are 
exponentially small. The latter reduces to

$$
\operatorname{Ai}^{\prime}\left(\xi_{0}-\xi_{a} / 2\right)=0
$$

for the $E_{r}$ wave, and to

$$
\operatorname{Ai}\left(\xi_{0}-\xi_{a} / 2\right)=0
$$

for the $E_{z}$ wave. The argument of the Airy function in Eqs. (23a) and (23b) is a large negative number and we can use the asymptotic formula

$$
\operatorname{Ai}(\xi) \approx \frac{1}{\sqrt{\pi}(-\xi)^{1 / 4}} \sin \left[\frac{2}{3}(-\xi)^{3 / 2}+\frac{\pi}{4}\right],
$$

which is actually valid with high accuracy for $\xi \lesssim-1$. We obtain

$$
\xi_{0} \approx \frac{\xi_{a}}{2}-\left[\frac{3 \pi}{2}\left(m \pm \frac{1}{4}\right)\right]^{2 / 3},
$$

where the upper sign corresponds to the $E_{r}$ mode while the lower one corresponds to the $E_{z}$ mode. The radial mode number $m$ runs over values $1,2,3, \ldots$ for the $E_{r}$ mode and $0,1,2, \ldots$ for the $E_{z}$ mode.

Using the definitions of $\xi_{a}$ and $\xi_{0}$, Eq. (16), we find

$$
\Lambda=\frac{\pi^{2} p^{2}}{2 k^{2} b^{2}}+\frac{1}{2}\left[\frac{3 \pi}{k R}\left(m \pm \frac{1}{4}\right)\right]^{2 / 3}-\frac{a}{2 R} .
$$

Eigenfunctions of toroidal modes in this limit can also be simplified:

$$
E_{r}=\operatorname{Ai}\left(\xi_{0}-\xi\right)
$$

for the $E_{r}$ mode, and

$$
E_{z}=\operatorname{Ai}\left(\xi_{0}-\xi\right)
$$

for the $E_{z}$ one. The modes are evanescent in the inner part of the camera, closer to the axis of the toroid, where the argument $\xi_{0}-\xi$ of the Airy function is positive. The modes oscillate in the outer part of the toroidal cross section.

Solving Eqs. (20) and (25) together, we find the function $F_{m}$ in Eq. (21):

$$
F_{m}(\zeta)=\frac{\pi \zeta}{\sqrt{2}} \mathcal{F}\left[\frac{2}{3}\left(\frac{m \pm 1 / 4}{\zeta}\right)^{2}\right]
$$

where

$$
\begin{aligned}
\mathcal{F}(q)= & \left\{q^{1 / 3}+[1+q-\sqrt{1+2 q}]^{1 / 3}\right. \\
& \left.+[1+q+\sqrt{1+2 q}]^{1 / 3}\right\}^{3 / 2}
\end{aligned}
$$

Approximate solution (28) is shown in Fig. 2 by dashed lines. It is very close to the exact solution Eq. (21), shown in solid lines, everywhere except for the $E_{r}, m=0$ modes in the case $p a / b \leqq 1$ (the lowest curve in Fig. 2), where a more accurate treatment reveals that $F_{0}(\zeta)=30^{1 / 4} \sqrt{\pi \zeta}$.

To prove that the condition Eq. (22) is satisfied at high frequencies we will show that it is satisfied if either $m$ or $p$ (or both) are much greater that unity. Since $\xi_{0}>0$, we need to prove that $\xi_{a} \gg 1$; for simplicity, we will assume that $a \sim b$. For $m \gg 1$, from Eq. (25) it follows that $\xi_{a} \gtrsim$ $m^{2 / 3}$, and indeed $\xi_{a}$ is large for large $m$. If, however, $m \sim 1$, then it follows from Eq. (25) that $\xi_{a} \sim \xi_{0}$, which together with Eq. (20) gives $\xi_{a} \sim p^{2 / 3}$, and again $\xi_{a}$ is large when $p \gg 1$. Hence the result of this subsection is applicable to modes that have at least one eigennumber much larger than unity.

Remarkably, approximate formulas of this section give accurate numerical results even at the edge of the applicability region, specified by Eq. (22). For example, Eq. (28) yields $F_{m}(1)=5.00$, which differs by $4.4 \%$ from the exact value 4.78 given by Eq. (21).

We note here that our solution can also be simplified in the limit where the condition opposite to Eq. (22) is satisfied. However, we will not consider this case here because the modes in this limit have phase velocities that are greater than the speed of light and cannot be excited by the beam.

\section{RADIATION OF A MOVING CHARGE}

In the preceding sections, we formulated equations whose solution gives synchronous eigenmodes propagating with the phase velocity close to the speed of light. A relativistic particle moving in a circular orbit inside the toroid will resonantly interact with these modes and will deposit part of its kinetic energy into the electromagnetic field of the modes. The strength of the interaction can be characterized by the amount of energy radiated into a given synchronous mode. As shown in Appendix B, the radiated energy per unit length of path $d W / d s$ is given by the following formula:

$$
\frac{d W}{d s}=\frac{e^{2}}{4 P}\left|\mathcal{E}_{0}\right|^{2}\left|\frac{1}{v_{g}}-\frac{1}{v}\right|^{-1},
$$

where $P$ is the averaged over time energy flow in the mode, $v_{g}$ is the group velocity, and $\mathcal{E}_{0}$ is the amplitude of the longitudinal electric field of the mode on the particle's trajectory. A related parameter, which is usually used in accelerator physics, is the loss factor $\kappa$ defined as the energy loss of a unit charge:

$$
\kappa=\frac{1}{e^{2}} \frac{d W}{d s}=\frac{1}{4 P}\left|\mathcal{E}_{0}\right|^{2}\left|\frac{1}{v_{g}}-\frac{1}{v}\right|^{-1} .
$$

From Eq. (29) we see that in order to calculate the radiated energy for each mode one has to know the energy flow $P$ and the group velocity of the mode $v_{g}$. The former can be expressed as an integral over the cross section of the chamber of the component of the Poynting vector $S_{\theta}$ along the direction of propagation,

$$
P=\int S_{\theta} d x d z
$$


where

$$
S_{\theta}=\frac{c}{8 \pi}\left(-E_{r} H_{z}+E_{z} H_{r}\right)=\frac{c}{8 \pi}\left(E_{r}^{2}+E_{z}^{2}\right),
$$

and we took into account that the averaging over time introduces a factor of $1 / 2$ in $S_{\theta}$. The quantity $P$ can be easily calculated by numerical integration if the fields are available.

Calculation of the group velocity using the definition $v_{g}=\partial \omega / \partial k$ requires numerical differentiation of the dispersion relation. There is however an alternative method of calculation of $v_{g}$, more suitable for numerical analysis. We recall that in a waveguide with straight axis the group velocity is equal to the ratio of energy flux throughout the cross section of the waveguide to the energy density integrated over the cross section. In toroid, this relation takes the form

$$
v_{g}=\frac{\int S_{\theta} d x d z}{\int w(1+x / R) d x d z},
$$

where

$$
w=\frac{1}{16 \pi}\left(E^{2}+H^{2}\right)
$$

is the averaged over time of the energy density in the wave. The geometric factor $1+x / R$ in Eq. (33) takes into account that the infinitesimal volume in the toroid is equal to $(1+x / R) d x d z d s$. The expression for $v_{g}$ in terms of the fields $E_{r}$ and $E_{z}$, which follows from Eqs. (33) and (34) is obtained in Appendix C:

$$
\frac{v_{g}}{c}=1-\frac{2}{R} \frac{\int d x d z x\left(E_{r}^{2}+E_{z}^{2}\right)}{\int d x d z\left(E_{r}^{2}+E_{z}^{2}\right)}-\Lambda .
$$

Equations (31), (32), and (35) give explicit expressions for the energy radiated into an eigenmode in terms of the field components $E_{r}, E_{z}$ and the longitudinal electric field on the axis $\mathcal{E}_{0}$.

\section{Wake and impedance}

A longitudinal impedance $Z(\omega)$ generated by the radiation of a point charge in a single mode has a resonant character. In the vicinity of the resonance it can be described by the following formula [3]:

$$
Z(\omega)=\frac{i \kappa}{\left(\omega-\omega_{s}\right)+i \Gamma / 2},
$$

where $\Gamma$ is the resonance width associated with the finite conductivity of the wall and $\kappa$ is the loss factor. In the limit of a perfectly conducting wall, which we consider here, $\Gamma \rightarrow 0$, and

$$
Z(\omega)=\kappa \pi \delta\left(\omega-\omega_{s}\right)+i \kappa \mathcal{P} \frac{1}{\omega-\omega_{s}},
$$

where $\mathcal{P}$ denotes the Cauchy principal part. This impedance corresponds to the following wake function $w(s)$ (see, e.g., [7]):

$$
w(s)=2 \kappa \cos \frac{\omega_{s} s}{c},
$$

which simply means that the charge excites the synchronous wave with the amplitude of the longitudinal electric field in the wave equal to $2 \kappa e^{2}$.

\section{RADIATION IN RECTANGULAR AND CIRCULAR TOROIDS}

In this section we will present results of numerical calculations of the mode patterns, frequencies, and loss factors for several lowest modes in the toroids of rectangular and round cross sections. In these calculations we assume $\Lambda=0$, corresponding to the phase velocity of waves equal to the speed of light.

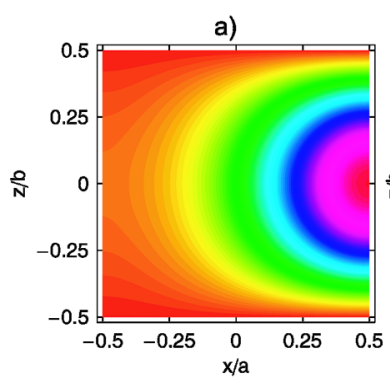

c)

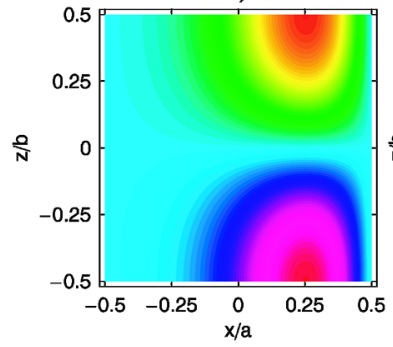

e)

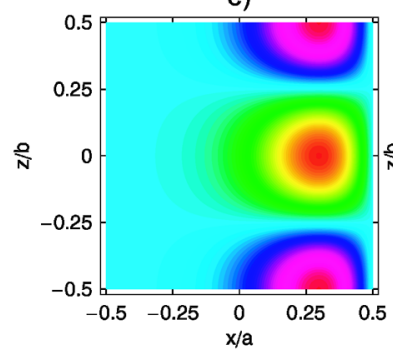

b)

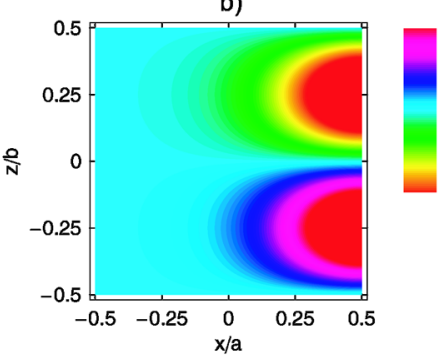

d)

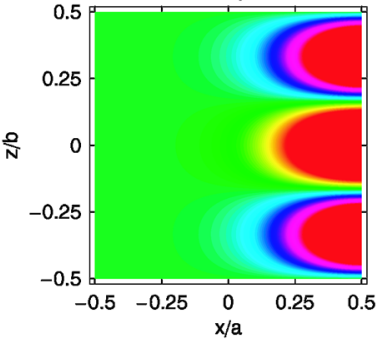

f)

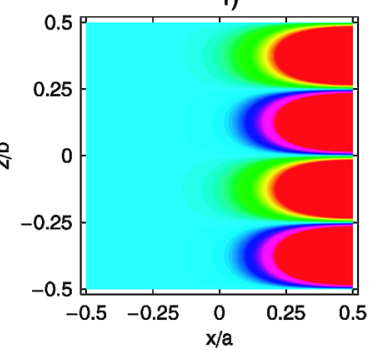

FIG. 3. (Color) Field patterns for a square cross section of the waveguide: (a) $E_{r}$ mode, $m=0, p=1$; (b) $E_{r}$ mode, $m=0$, $p=2$; (c) $E_{z}$ mode, $m=1, p=1$; (d) $E_{r}$ mode, $m=0, p=3$; (e) $E_{z}$ mode, $m=1, p=2$; (f) $E_{r}$ mode, $m=0, p=4$. For the $E_{z}$ modes, the graphs show distribution of the $E_{z}$ component of the field; and for the $E_{r}$ modes, the $E_{r}$ distribution is shown. The color coding palette is shown in the upper right corner of the picture, where the bottom and the top of the palette correspond to lower and higher values, respectively. Note that the mode profiles are not normalized, and the patterns of different modes cannot be compared with each other. 
Calculations for the rectangular cross section are based on the analytical solution developed in Sec. IV. For the sake of simplicity, we only consider here the case of the square cross section, $a=b$.

We remind one that in this case there are two uncoupled types of modes $-E_{r}$ and $E_{z}$ - which are characterized by the integer numbers $m$ and $p$, where $p$ is defined in Eqs. (15a) and (15b) and $m$ is equal to the number of zeros in the function $U(\xi)$ [see Eq. (17)]. The field patterns for a few lowest modes are shown in Fig. 3 and the frequency, group velocity, and the loss factors for each mode are given in Table I. The lowest mode in this case is of type $E_{r}$, with the frequency equal to $4.78 c R^{1 / 2} a^{-3 / 2}$ and the loss factor $\kappa \approx 5 / a^{2}$.

To calculate the eigenmodes for the round cross section we used two approaches. In the first one, the system of equations for $E_{r}$ and $E_{z}$ was solved numerically on a triangular mesh, using the PDE Toolbox of the Matlab [8]. The accuracy of the numerical solution depends on the number of triangles $N$; we evaluated that it scales as $\left(m^{2}+p^{2}\right) / N$. The maximum number of $N$ was limited by the run time which grows as $N^{2}$ - we typically used $N \approx 10^{4}$ at most.

The second approach was based on the variational principle described in Appendix A. In this approach, the solution was represented as a sum of trial functions with unknown coefficients, and the coefficients were calculated by minimizing the functional $I$. The results of both methods agree within $10 \%$ with each other. In Table II we show the frequency, group velocity, and the

TABLE I. Lowest modes in the toroidal waveguide of the square cross section. The wave number of the mode $k$, the difference between $c$ and the group velocity, $1-v_{g} / c$, and the loss factory $\kappa$ are all normalized by the proper combinations of $R$ and $a$.

\begin{tabular}{cccccl}
\hline \hline Type & $m$ & $p$ & $k R^{-1 / 2} a^{3 / 2}$ & $\left(1-v_{g} / c\right) R / a$ & $\kappa a^{2}$ \\
\hline$E_{r}$ & 0 & 1 & 4.78 & 0.62 & 4.94 \\
$E_{r}$ & 0 & 2 & 8.11 & 0.73 & 0 \\
$E_{z}$ & 1 & 1 & 8.78 & 0.42 & 3.01 \\
$E_{r}$ & 0 & 3 & 11.42 & 0.79 & 0.19 \\
$E_{z}$ & 1 & 2 & 11.80 & 0.52 & 0 \\
\hline \hline
\end{tabular}

TABLE II. The lowest modes in a toroidal waveguide with the round cross section. The notations are the same as in Table I.

\begin{tabular}{ccl}
\hline \hline$k R^{-1 / 2} a^{3 / 2}$ & $\left(1-v_{g} / c\right) R / a$ & $\kappa a^{2}$ \\
\hline 2.12 & 1.08 & 2.11 \\
2.73 & 0.79 & 0 \\
3.96 & 0.88 & 0.33 \\
4.07 & 0.96 & 0 \\
4.82 & 0.76 & 1.04 \\
\hline \hline
\end{tabular}

loss factors for a few lowest modes. Note that the modes in which $E_{z}$ is an even and $E_{r}$ is an odd function of $z$ have a zero loss factor-this is explained by the fact that for these modes, as follows from Eq. (7), the longitudinal electric field vanishes at the location of the particle $z=0$.

The field patterns for the lowest three modes that have nonvanishing loss factors (the first, third, and fifth lines in Table II) are shown in Fig. 4.

The lowest modes with $m \sim p \sim 1$ correspond to the waves propagating at an angle $\theta \sim \sqrt{a / R}$ relative to the minor axis of the toroid. Modes with $m, p \gg 1$ propagate at smaller angles, and during the process of radiation of such modes, they do not "feel" the presence of the conducting boundaries of the waveguide. In this limit, we expect that the spectral density of the radiation approaches that in free space, and, hence, does not depend on the exact shape of the cross section of the chamber. For the case of a rectangular cross section we prove this

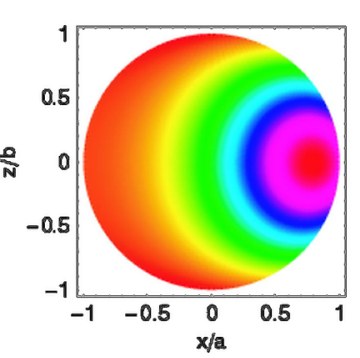

a)

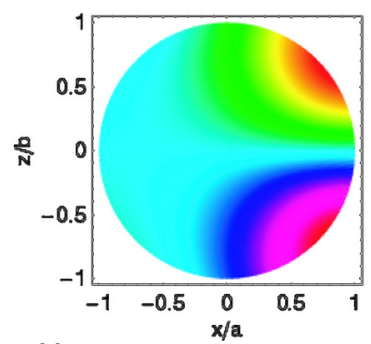

b)
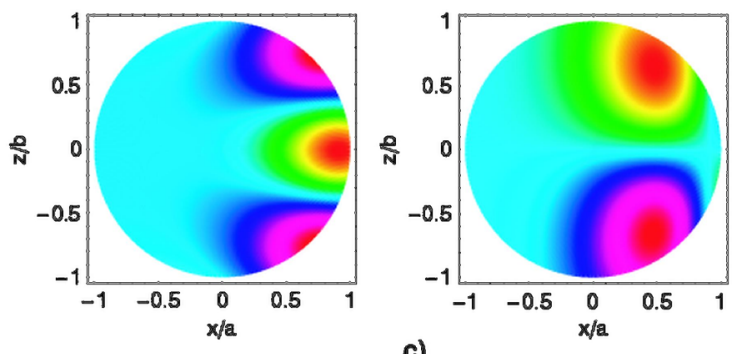

c)
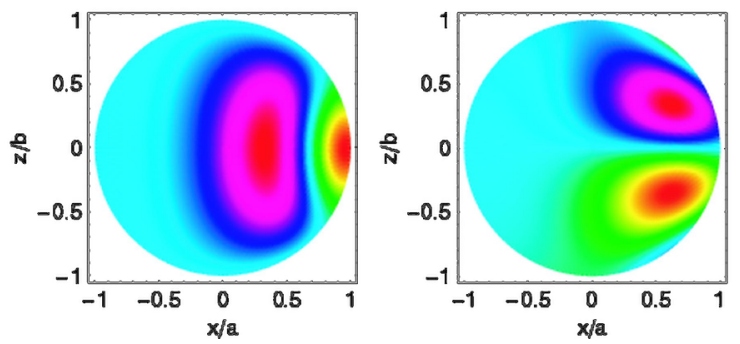

FIG. 4. (Color) Field profiles for a round cross section of the waveguide for the three lowest antisymmetric modes: (a) $k R^{-1 / 2} a^{3 / 2}=2.12$; (b) $k R^{-1 / 2} a^{3 / 2}=3.95$; (c) $k R^{-1 / 2} a^{3 / 2}=$ 4.82. The left plot in each pair of graphs shows distribution of the $E_{r}$ in the mode, and the right one shows the distribution of $E_{z}$. The color coding palette is shown in the upper right corner of the picture. 
assertion in Appendix D by direct calculation of the spectrum of the radiation in the limit $\omega \gg \omega_{\max }$.

\section{CONCLUSIONS}

In this paper we developed a new approach to the calculation of the synchrotron radiation in a toroidal pipe. Using a small parameter $\epsilon=\sqrt{a / R}$ we simplified Maxwell's equations assuming that the characteristic frequency of the modes $\omega \sim c / a \epsilon$ and neglected terms of higher order if $\epsilon$. The resulting equations involve only two components of the field $E_{r}$ and $E_{z}$. For a rectangular cross section of the waveguide, we found an analytical solution of the equations and analyzed its asymptotics at very high frequency.

Based on Vainshtein's formulation of the radiation problem, we then obtained an equation which gives radiation into each synchronous mode. The energy loss involves three characteristics of the mode: the group velocity, the longitudinal electric field on the orbit, and the energy flow in the mode. We showed how all these three quantities can be expressed in terms of $E_{z}$ and $E_{r}$ in a form that allows an easy numerical evaluation.

Finally, we demonstrated the flexibility of the new method by calculating the frequencies and the loss factors for the lowest modes in square and round waveguides.

\section{ACKNOWLEDGMENTS}

The authors would like to acknowledge the valuable contribution of Dr. V. S. Cherkassky in the development of the Matlab code. This work was supported by the Department of Energy, Contract No. DE-AC0376SF00515

\section{APPENDIX A: VARIATIONAL PRINCIPLE}

In this Appendix we will show how Eqs. (10) with the boundary conditions (12a) and (12b) can be cast into a variational principle.

Let us introduce the two-dimensional vector $\boldsymbol{w}, \boldsymbol{w}=$ $\boldsymbol{e}_{x} E_{z}-\boldsymbol{e}_{z} E_{r}$, where $\boldsymbol{e}_{x}$ and $\boldsymbol{e}_{z}$ are unit vectors in $x$ and $z$ directions. From Eqs. (10) we find that $\boldsymbol{w}$ satisfies the following equation:

$$
\Delta w+\lambda x^{\prime} w=0
$$

where $\lambda=2 k^{2} / R$, and $x^{\prime}=x+R \Lambda$. The boundary condition (12b) means that $\boldsymbol{w}$ has only a tangential component on the boundary,

$$
\boldsymbol{w} \cdot \boldsymbol{n}=0
$$

The boundary condition (12a) reads

$$
\operatorname{rot} w=0 .
$$

If we write Eq. (A1) as

$$
\hat{K} w=0,
$$

where $\hat{K}$ is a linear operator, in order to be able to prove a variational principle, we need to show that the operator $\hat{K}$ is symmetric:

$$
\int d S \boldsymbol{u} \cdot \hat{K} \boldsymbol{w}=\int d S \boldsymbol{w} \cdot \hat{K} \boldsymbol{u}
$$

where the functions $\boldsymbol{u}, \boldsymbol{w}$ satisfy the boundary conditions (A2) and (A3). To prove the symmetry of $\hat{K}$, we introduce a functional $I$

$$
I=-\int d S \boldsymbol{u} \cdot \hat{K} \boldsymbol{w}=-\int d S\left(\boldsymbol{u} \cdot \Delta \boldsymbol{w}+\lambda x^{\prime} \boldsymbol{u} \cdot \boldsymbol{w}\right) .
$$

Substituting the vector identities

$$
\begin{aligned}
\mathbf{u} \cdot \Delta \boldsymbol{w}= & \boldsymbol{u} \operatorname{grad} \operatorname{div} \boldsymbol{w}-\boldsymbol{u \operatorname { r o t } \operatorname { r o t } w} \\
= & \operatorname{div}(\boldsymbol{u} \operatorname{div} w)-\operatorname{div} \boldsymbol{u} \operatorname{div} \boldsymbol{w}+\operatorname{div}(\boldsymbol{u} \times \operatorname{rot} w) \\
& -\operatorname{rot} u \operatorname{rot} w
\end{aligned}
$$

into Eq. (A6), and performing the integration we find that the div terms vanish because of the boundary conditions (A2) and (A3), so that

$$
I=\int d S\left(\operatorname{rot} \boldsymbol{u} \cdot \operatorname{rot} \boldsymbol{w}+\operatorname{div} \boldsymbol{u} \cdot \operatorname{div} \boldsymbol{w}-\lambda x^{\prime} \boldsymbol{u} \cdot \boldsymbol{w}\right) .
$$

This expression is clearly symmetric with respect to exchange $\boldsymbol{w} \leftrightarrow \boldsymbol{u}$, which proves the relation Eq. (A5).

To obtain the variational principle, we set $\boldsymbol{u}=\boldsymbol{w}$ :

$$
I=\int d S\left[(\operatorname{rot} w)^{2}+(\operatorname{div} \boldsymbol{w})^{2}-\lambda x^{\prime} w^{2}\right] .
$$

It is now easy to see that the condition $\delta I=0$, for the functions $w$ that satisfy the boundary conditions Eqs. (A2) and (A3), constitutes a variation principle for the Eq. (A1).

It follows from Eq. (A6) that if $\boldsymbol{w}$ is a solution of Eq. (A1) with the eigenvalue $\lambda$, then $I=0$. Using now Eq. (A9), we can express the eigenvalue $\lambda$ through integrals of the solution $w$ :

$$
\begin{aligned}
\lambda & =\frac{2 k^{2}}{R}=\frac{\int d S\left[(\operatorname{rot} w)^{2}+(\operatorname{div} \boldsymbol{w})^{2}\right]}{\int d S x^{\prime} w^{2}} \\
& =\frac{\int d x d z\left[\left(\frac{\partial E_{z}}{\partial z}+\frac{\partial E_{r}}{\partial x}\right)^{2}+\left(\frac{\partial E_{r}}{\partial z}-\frac{\partial E_{z}}{\partial x}\right)^{2}\right]}{\int d x d z(x+R \Lambda)\left(E_{r}^{2}+E_{z}^{2}\right)} .
\end{aligned}
$$

This relation turns out useful in the calculation of the group velocity of eigenmodes; see Appendix C.

\section{APPENDIX B: RADIATION OF A RELATIVISTIC CHARGE MOVING IN TORODIDAL CHAMBER}

Let us consider a point charge moving with relativistic velocity in the toroidal vacuum chamber along the axis of the toroid. To calculate the energy radiated by the charge into synchronous modes we will use the approach developed by Vainshtein [9] (see also [5]). This approach gives an explicit expression for the amplitudes of the modes 
excited by an arbitrary distribution of current $\boldsymbol{j}(\boldsymbol{r}) e^{-i \omega t}$ oscillating with the frequency $\omega$.

Let the index $w$ denote an eigenmode with the frequency $\omega$ propagating in the direction of the particle's motion (the direction in which $\theta$ increases), and $-w$ denotes the same mode propagating in the opposite direction. The electric field of the eigenmode $w$ is $\boldsymbol{E}_{w}(\boldsymbol{r}) e^{-i \omega t}$. An external current $\boldsymbol{j}(\boldsymbol{r}) e^{-i \omega t}$ in the waveguide excites this mode with an amplitude $C_{w}(s)$ so that the electric field $\boldsymbol{E}$ in the mode is

$$
\mathbf{E}(\boldsymbol{r})=C_{w}(s) \boldsymbol{E}_{w}(\boldsymbol{r}),
$$

where $s=R \theta$ is the coordinate along the axis of the toroid. The formula for $C_{w}$ reads [9]

$$
C_{w}(s)=\frac{1}{N_{w}} \int_{-\infty}^{s} d s d S \boldsymbol{j}(\boldsymbol{r}) \cdot \boldsymbol{E}_{-w}(\boldsymbol{r}),
$$

where $\boldsymbol{E}_{-w}$ is the electric field of the eigenmode propagating in the opposite direction, $N_{w}$ is the norm of the mode,

$$
N_{w}=\frac{c}{4 \pi} \int d S \boldsymbol{e}_{\theta} \cdot\left(\boldsymbol{E}_{w} \times \boldsymbol{H}_{-w}-\boldsymbol{E}_{-w} \times \boldsymbol{H}_{w}\right),
$$

the integral in Eq. (B2) is taken over the volume to the left of the point at which $C_{w}(s)$ is evaluated, and the integral in Eq. (B3) is taken over the cross section of the waveguide. The vector $\boldsymbol{e}_{\theta}$ in Eq. (B3) is a unit vector in the $\theta$ direction. We will assume that the fields in the modes $w$ and $-w$ are chosen so that $\boldsymbol{E}_{-w}=-\boldsymbol{E}_{w}^{*}$, then one can show that the norm is equal to 4 times the averaged over time of the energy flow $P_{w}$ in the mode, $N_{w}=4 P_{w}$ [9]. The field $\boldsymbol{E}_{-w}$ can be represented as

$$
\boldsymbol{E}_{-w}(\boldsymbol{r})=-\mathcal{E}_{w}^{*}(x, z) e^{-i k(\omega) s},
$$

where $\mathcal{E}_{w}(x, z)$ gives the transverse distribution of the electric field in the $w$ eigenmode in the plane $x, z$, and $k(\omega)$ is the wave number as a function of the frequency.

We now calculate the Fourier components of the current corresponding to the point charge moving with velocity $v$. The current density has only the $\theta$ component

$$
j_{\theta}(\boldsymbol{r}, t)=e v \delta(x) \delta(z) \delta(s-v t) .
$$

Making the Fourier transformation of the current Eq. (B5) yields

$$
\frac{1}{2 \pi} \int d t j_{\theta}(\boldsymbol{r}, t) e^{i \omega t}=\frac{e}{2 \pi} \delta(x) \delta(z) e^{i \omega s / v} .
$$

Inserting this expression into Eq. (B2) gives the following result for the amplitude $C_{w}$ :

$$
\begin{aligned}
C_{w}(s) & =\frac{e}{2 \pi N_{w}} \mathcal{E}_{0} \int_{-\infty}^{s} d s^{\prime} e^{-i s^{\prime}[k(\omega)-\omega / v]} \\
& =\frac{i e \mathcal{E}_{0}}{2 \pi N_{w}} \frac{e^{-i s[k(\omega)-\omega / v]}}{k(\omega)-\frac{\omega}{v}+i 0},
\end{aligned}
$$

where $\mathcal{E}_{0}=-\mathcal{E}_{w, s}^{*}(0,0)=\mathcal{E}_{w, s}(0,0)$ is the longitudinal electric field of the eigenmode on the particle's path and we took into account that $\mathcal{E}_{0}$ is purely imaginary in our problem [see Eq. (7)]. As seen from Eq. (B7), the function $C_{w}$ has a singularity at the frequency of the synchronous mode $\omega_{s}$ that satisfies the equation

$$
\omega_{s}=v k\left(\omega_{s}\right)
$$

The term $i 0$ in the denominator of Eq. (B7) indicates an infinitesimally small imaginary part that introduces a shift of the pole from the real axis - in the general case the pole can be shifted either up or down from the real axis $\omega$.

Let us now calculate the energy $W$ radiated by the particle per unit time into the synchronous mode. In what follows, we will assume that the particle's velocity $v \approx c$ is greater than the group velocity of the mode $v_{g}$. We will drop this assumption at the end of derivation.

First, we find the longitudinal electric field $E(s, t)$ on the axis by making the inverse Fourier transform of the electric field. Note that $k(\omega)$ is an odd function of $\omega$, hence there are always two solutions for $\omega_{s}$ with opposite signs. Using Eq. (B7) we find

$$
\begin{aligned}
E(s, t) & =\int_{-\infty}^{\infty} d \omega C_{w} \mathcal{E}_{0} e^{i k(\omega) s-i \omega t} \\
& =\frac{i e \mathcal{E}_{0}^{2}}{2 \pi N_{w}} \int_{-\infty}^{\infty} d \omega \frac{e^{i s \omega / v-i \omega t}}{k(\omega)-\frac{\omega}{v}+i 0} .
\end{aligned}
$$

Expanding the denominator in the integrand near the pole,

$$
\begin{aligned}
k(\omega)-\frac{\omega}{v}+i 0 & \approx\left(\omega-\omega_{s}\right)\left(\left.\frac{d k}{d \omega}\right|_{\omega=\omega_{s}}-\frac{1}{v}\right)+i 0 \\
& =\left(\omega-\omega_{s}\right)\left(\frac{1}{v_{g}}-\frac{1}{v}\right)+i 0,
\end{aligned}
$$

where $v_{g}$ is the group velocity of the mode, $v_{g}=$ $d \omega /\left.d k\right|_{\omega=\omega_{s}}$, it is easy to see that in the case when $v>v_{g}$ the pole is located below the real axis $\omega$. In front of the particle, where $s>v t$, we can close the integration path in the integral of Eq. (B9) by an infinite half circle in the upper plane of $\omega$, and since there are no poles inside such an integration contour, the integral vanishes. Hence the field in front of the particle is equal to zero.

The field behind the particle, $s<v t$, can be obtained by shifting the integration path below the real axis, $\operatorname{Im} \omega<0$. The contribution from the poles should be interpreted as a radiation field associate with the synchronous modes. It is easy to find this contribution by calculating the two residues as $\omega= \pm \omega_{s}$,

$$
E(s, t)=\frac{2 e \mathcal{E}_{0}^{2}}{N_{w}}\left(\frac{1}{v_{g}}-\frac{1}{v}\right)^{-1} \cos \left[\frac{\omega_{s}}{v}(s-v t)\right] .
$$

As might be expected, the field behind the particle is a 
sinusoidal oscillation with the frequency and the wave number equal to those of the synchronous mode $w$.

Since the electric field in front of the particle is zero, the effective electric field that acts on the charge is equal to half of the field behind it, $E_{\text {eff }}=\frac{1}{2} E(s=v t-0)$. The energy lost by the particle per unit length of the path can be calculated as a work of the force $e E_{\text {eff }}$ :

$$
\frac{d W}{d s}=-e E_{\text {eff }}=\frac{e^{2}}{N_{w}}\left|\mathcal{E}_{0}\right|^{2}\left(\frac{1}{v_{g}}-\frac{1}{v}\right)^{-1} .
$$

Recalling that $N_{w}=4 P_{w}$, for the ultrarelativistic particle, we arrive at Eq. (29) (the index $w$ is suppressed for ease of notation).

If $v<v_{g}$, the above derivation remains valid with minor amendments. It is readily seen that in this case the wake field is located in front of the particle, and the formula (B12) is still valid if one substitutes the last factor $\left(1 / v_{g}-1 / v\right)^{-1}$ with its absolute value $\left|1 / v_{g}-1 / v\right|^{-1}$.

\section{APPENDIX C: AN EXPRESSION FOR $\boldsymbol{v}_{g}$}

We will see below that the difference $1-v_{g} / c$ is of the second order in parameter $\epsilon$ and it might seem that in order to calculate $1-v_{g} / c$ one has to know the fields up to the second order. Fortunately, it turns out that the second order terms cancel out and the final result is formulated in terms of the zeroth and first order terms only.

Recall that $E_{z}, H_{z}, E_{r}$, and $H_{r}$ are of the zeroth order, and $E_{\theta}$ and $H_{\theta}$ are of the first order in $\epsilon$. Suppose that we know the functions $E_{z}, H_{z}, E_{r}$, and $H_{r}$ up to the terms of the order of $\epsilon^{2}$, i.e.,

$$
\begin{array}{ll}
E_{z}=E_{z}^{(0)}+E_{z}^{(2)}, & H_{z}=H_{z}^{(0)}+H_{z}^{(2)}, \\
E_{r}=E_{r}^{(0)}+E_{r}^{(2)}, & H_{r}=H_{r}^{(0)}+H_{r}^{(2)},
\end{array}
$$

where the superscript indicates the order in $\epsilon$. The terms of the second order in the $\theta$ component of the Poynting vector $S_{\theta}$, Eq. (32), will be

$$
S_{\theta}=\frac{c}{8 \pi}\left(-E_{r}^{(0)} H_{z}^{(2)}-E_{r}^{(2)} H_{z}^{(0)}+E_{z}^{(0)} H_{r}^{(2)}+E_{z}^{(2)} H_{r}^{(0)}\right),
$$

and the terms of the second order in $w$ will be

$$
\begin{aligned}
w=\frac{1}{8 \pi}\left(E_{z}^{(0)} E_{z}^{(2)}+E_{r}^{(0)} E_{r}^{(2)}+H_{z}^{(0)} H_{z}^{(2)}+H_{r}^{(0)} H_{r}^{(2)}\right. \\
\left.+E_{\theta}^{2}+H_{\theta}^{2}\right) .
\end{aligned}
$$

Using Eqs. (5) and (7) for the zero-order components of the fields and substituting Eqs. (C1) and (C2) into Eq. (33) we find that the second order terms will cancel each other:

$$
\begin{aligned}
\frac{v_{g}}{c}= & 1-\frac{1}{2 k^{2}} \frac{\int d x d z\left[\left(\frac{\partial E_{z}}{\partial z}+\frac{\partial E_{r}}{\partial x}\right)^{2}+\left(\frac{\partial E_{r}}{\partial z}-\frac{\partial E_{z}}{\partial x}\right)^{2}\right]}{\int d x d z\left(E_{r}^{2}+E_{z}^{2}\right)} \\
& -\frac{1}{R} \frac{\int d x d z x\left(E_{r}^{2}+E_{z}^{2}\right)}{\int d x d z\left(E_{r}^{2}+E_{z}^{2}\right)} .
\end{aligned}
$$

Further simplification of this expression is possible by applying the variational principle [(A10)]. According to this principle, the second term on the right-hand side is equal to the third one plus $\Lambda$, so that

$$
\frac{v_{g}}{c}=1-\frac{2}{R} \frac{\int d x d z x\left(E_{r}^{2}+E_{z}^{2}\right)}{\int d x d z\left(E_{r}^{2}+E_{z}^{2}\right)}-\Lambda
$$

\section{APPENDIX D: RADIATION IN THE LIMIT OF HIGH FREQUENCIES}

When the frequency of the radiation $\omega$ is much larger than the critical frequency for the toroidal waveguide $\omega \gg \omega_{\min }=c k_{\min }$ [see Eq. (1)], the shielding effect of the conducting boundaries becomes small. One expects that in this limit the spectrum of the radiation does not depend on the shape of the boundary and, with increase of $\omega$, approaches the spectrum of the synchrotron radiation in vacuum. In this Appendix, we will demonstrate, by direct calculation for the rectangular cross section, that the limit $\omega \rightarrow \infty$ recovers the well-known formulas for the synchrotron radiation in free space.

Since the radiation in free space has a continuous spectrum, we expect that in the limit of large $\omega$ the main contribution to the radiated energy will come from modes with $p, m \gg 1$ (see the subsection of IV). This means that we can neglect the addend $\frac{1}{4}$ in comparison with $m, m \pm \frac{1}{4} \rightarrow m$, in Eqs. (25) and (26).

Let us first do calculations for the $E_{z}$ mode; the contribution from the $E_{r}$ mode is similar and its derivation is outlined at the end of the Appendix. First we calculate the electric field $\mathcal{E}_{0}$ on the axis,

$$
\mathcal{E}_{0}=\left.E_{\theta}\right|_{x, z=0}=\left.\frac{i}{k} \frac{\partial E_{z}}{\partial z}\right|_{x, z=0}=-\left.\frac{i \pi p}{k b} \sin \left(\frac{\pi p}{2}\right) E_{z}\right|_{\xi=0},
$$

which tells that it vanishes for even $p$; for odd $p$ we have

$$
\left|\mathcal{E}_{0}\right|=\frac{\pi p}{k b}\left|E_{z}\right|_{\xi=0}=\frac{\pi p}{k b}\left|\operatorname{Ai}\left(\xi_{0}\right)\right|,
$$

where we used Eq. (27b).

The energy radiated per unit path of the particle is given by Eq. (B12):

$$
\frac{d W}{d s}=\sum_{p, m} \frac{e^{2}}{4 P}\left|\mathcal{E}_{0}\right|^{2}\left|\frac{1}{v_{g}}-\frac{1}{v}\right|^{-1},
$$

where $\mathcal{E}_{0}, P$, and $v_{g}$ are functions of $p$ and $m$. Since we know that large values of $p$ and $m$ make the dominant 
contribution, we will use integration instead of summation. Also, since only odd $p$ contributes, we will add a factor $1 / 2$ :

$$
\begin{aligned}
\frac{d W}{d s} & =\frac{1}{8} e^{2} \int_{0}^{\infty} d m \int_{0}^{\infty} d p \frac{1}{P}\left|\mathcal{E}_{0}\right|^{2}\left|\frac{1}{v_{g}}-\frac{1}{v}\right|^{-1} \\
& =\frac{1}{8} e^{2} \int_{0}^{\infty} d m \int_{0}^{\infty} d p \int_{0}^{\infty} d k \frac{1}{P}\left|\mathcal{E}_{0}\right|^{2}\left|\frac{1}{v_{g}}-\frac{1}{v}\right|^{-1} \delta\left(k \Lambda+k / 2 \gamma^{2}\right)\left|\frac{d}{d k}\left(k \Lambda+\frac{k}{2 \gamma^{2}}\right)\right| .
\end{aligned}
$$

In the last equation we introduced integration over the wave number $k$ together with the delta function, which takes explicitly into account the resonance conditions $\Lambda=-1 / 2 \gamma^{2}$; the integration goes over the positive half of the $k$ axis because Eq. (B12) already takes into account modes propagating in opposite directions. Making use of Eqs. (3) and (25) one can show that

$$
\left|\frac{1}{v_{g}}-\frac{1}{v}\right|^{-1}\left|\frac{d}{d k}\left(k \Lambda+\frac{k}{2 \gamma^{2}}\right)\right|=\frac{v v_{g}}{c} \approx c
$$

which simplifies Eq. (D4)

$$
\frac{d W}{d s}=\frac{1}{8} c e^{2} \int d m \int d p \int d k \frac{1}{P}\left|\mathcal{E}_{0}\right|^{2} \delta\left(k \Lambda+k / 2 \gamma^{2}\right)
$$

Using Eq. (27b) and taking into account that in the $E_{z}$ mode $H_{r}=E_{z}$ and $H_{z}=E_{r}=0$, as follows from Eq. (5), it is easy to find the energy flow $P$ in the mode:

$$
P=\frac{c b}{16 \pi^{2}}\left(\frac{3 \pi m R}{4 k^{2}}\right)^{1 / 3}\left(1+\delta_{p 0}\right)
$$

Now substituting Eqs. (D2), (D6), and (26) into Eq. (D5) we obtain

$$
\frac{d W}{d s}=\frac{2^{2 / 3} \pi^{11 / 3}}{3^{1 / 3}} \frac{e^{2}}{b^{3} R^{1 / 3}} \int d m \int d p \int d k \frac{p^{2} E_{z}^{2}}{m^{1 / 3} k^{4 / 3}} \delta\left(k\left[-\frac{a}{2 R}+\frac{1}{2 \gamma^{2}}+\frac{\pi^{2} p^{2}}{2 k^{2} b^{2}}+\frac{1}{2}\left(\frac{3 \pi m}{k R}\right)^{2 / 3}\right]\right) .
$$

We first integrate over $p$ and get rid of the delta function

$$
\frac{d W}{d s}=\frac{2^{5 / 3} \pi^{5 / 3}}{3^{1 / 3}} \frac{e^{2}}{b R^{1 / 3}} \int d m \int d k \frac{p(m)}{m^{1 / 3} k^{1 / 3}} E_{z}^{2}
$$

where

$$
p(m)=\frac{k b}{\pi}\left[\frac{a}{R}-\frac{1}{\gamma^{2}}-\left(\frac{3 \pi m}{k R}\right)^{2 / 3}\right]^{1 / 2}
$$

Note that for a given $k, p$ varies from 1 to $p_{\max }=$ $(b k / \pi)\left(a / R-1 / \gamma^{2}\right)^{1 / 2}$ when $m$ varies from 0 to $m_{\max }=$ $(k R / 3 \pi)\left(a / R-1 / \gamma^{2}\right)^{3 / 2}$.

Equation (D7) is the energy radiated per unit length of the path. If we drop integration over $k$ from that equation and divide it by $v$, we obtain the energy loss per $d \omega=$ $v d k$, which is the spectral energy loss $P_{E}(\omega)$. A simple calculation yields

$$
\begin{aligned}
P_{E}= & \frac{2^{5 / 3} \pi^{5 / 3}}{(3 k R)^{1 / 3} c b} \\
& \times \int d m m^{-1 / 3} p(m) \mathrm{Ai}^{2}\left(\frac{\pi^{2} p^{2} R^{2 / 3}}{2^{2 / 3} k^{4 / 3} b^{2}}+\frac{\left(2 k^{2} R^{2}\right)^{1 / 3}}{2 \gamma^{2}}\right),
\end{aligned}
$$

where we used Eqs. (27a) and (27b) for $E_{z}$, Eq. (16) for $\xi_{0}$, and set $\Lambda=-1 / 2 \gamma^{2}, v=c$. Changing the integration variable from $m$ to $\mu$, with

$$
\mu=\frac{k^{2 / 3} R^{2 / 3}}{2^{2 / 3}}\left[\frac{a}{R}-\frac{1}{\gamma^{2}}-\left(\frac{3 \pi m}{k R}\right)^{2 / 3}\right]
$$

yields the following result:

$$
\begin{aligned}
P_{E}(\omega)= & \frac{2^{5 / 3} e^{2}}{c} k^{1 / 3} R^{-2 / 3} \\
& \times \int_{0}^{\infty} d \mu \sqrt{\mu} \mathrm{Ai}^{2}\left[\mu+\left(\frac{k R}{2 \gamma^{3}}\right)^{2 / 3}\right],
\end{aligned}
$$

where in the limit $a, b \rightarrow \infty$ the lower and upper limits of integration are zero and infinity, respectively.

So far we have found the radiation to $E_{z}$ modes. To calculate radiation $P_{H}$ to $E_{r}$ modes, it is sufficient to note that Eq. (D6) is also valid for $E_{r}$ modes (except for the $p=0$ mode which does not exist), $\partial E_{z} / \partial z$ in Eq. (D1) has to be substituted with $\partial E_{r} / \partial x$, and that $E_{r}$ in this limit is also equal to the Airy function, Eq. (27a). As a result, the expression for the spectral energy radiation $P_{H}(\omega)$ into $E_{r}$ modes is

$$
\begin{aligned}
P_{H}(\omega)= & \frac{2^{5 / 3} e^{2}}{c} k^{1 / 3} R^{-2 / 3} \\
& \times \int_{0}^{\infty} d \mu \frac{1}{\sqrt{\mu}} \mathrm{Ai}^{\prime 2}\left[\mu+\left(\frac{k R}{2 \gamma^{3}}\right)^{2 / 3}\right] .
\end{aligned}
$$

The total energy, radiated per unit path per unit frequency, is the sum of (D10) and (D11): 


$$
P(\omega)=\frac{e^{2} \omega_{H} \gamma}{c^{2}} 2^{5 / 3} \sqrt[3]{\frac{3 \zeta}{2}} \int_{0}^{\infty} d \mu\left\{\sqrt{\mu} \mathrm{Ai}^{2}\left[\mu+\left(\frac{3 \zeta}{4}\right)^{2 / 3}\right]+\frac{1}{\sqrt{\mu}} \mathrm{Ai}^{22}\left[\mu+\left(\frac{3 \zeta}{4}\right)^{2 / 3}\right]\right\}
$$

where $\zeta=2 k R / 3 \gamma^{3}$ and $\omega_{H}=c / R$.

One can show that Eq. (D12) can be cast into the form

$$
\begin{aligned}
& P(\omega)=\frac{e^{2} \omega_{H} \gamma}{c^{2}} F\left(\frac{2 k R}{3 \gamma^{3}}\right), \\
& F(\zeta)=\frac{\sqrt{3}}{2 \pi} \zeta \int_{\zeta}^{\infty} d \zeta K_{5 / 3}(\zeta) d \zeta,
\end{aligned}
$$

which coincides with Eq. (74.17) from [10].

[1] A.W. Chao and M. Tigner, Handbook of Accelerator Physics and Engineering (World Scientific, Singapore, 1999).

[2] S. Heifets and G.V. Stupakov, Phys. Rev. ST Accel. Beams 5, 054402 (2002).
[3] R. L. Warnock and P. Morton, Part. Accel. 25, 113 (1990).

[4] K.-Y. Ng, Part. Accel. 25, 153 (1990).

[5] M. M. Karliner, N.V. Mityanina, and V. P. Yakovlev, Budker Institute of Nuclear Physics Technical Report No. BUDKERINP 93-90, 1993.

[6] J. B. Murphy, S. Krinsky, and R. L. Gluckstern, Part. Accel. 57, 9 (1997).

[7] A.W. Chao, Physics of Collective Beam Instabilities in High-Energy Accelerators (Wiley, New York, 1993).

[8] Partial Differential Equation Toolbox User's Guide (The MathWorks, Inc., Natick, MA, 1995).

[9] L. A. Vainshtein, Electromagnetic Waves (Radio i svyaz', Moscow, 1988), in Russian.

[10] L. D. Landau and E. M. Lifshitz, The Classical Theory of Fields, Course of Theoretical Physics Vol. 2 (Pergamon, London, 1979), 4th ed., translated from the Russian. 